\section{ORIGINAL RESEARCH}

Z. Shao

B. Wang

Y. Wu

Z. Zhang

Q. Wu

S. Yang

\title{
CT-Guided Percutaneous Injection of Fibrin Glue for the Therapy of Symptomatic Arachnoid Cysts
}

BACKGROUND AND PURPOSE: Although often asymptomatic, enlarging sacral arachnoid cysts can also cause significant discomfort. These symptomatic sacral arachnoid cysts require specific treatments to facilitate local decompression. The main drawback of the surgical managements is the high risk of recurrence and complications. To decrease risks, we had been attempting to seek a simple and safe method to manage the symptomatic sacral arachnoid cysts. CT-guided percutaneous fibrin glue therapy is a new nonsurgical alternative. To evaluate the efficacy of the novel nonsurgical approach, we conducted this retrospective study of 38 patients.

MATERIALS AND METHODS: Thirty-eight patients with symptomatic sacral arachnoid cysts underwent fibrin glue therapy in our hospital between June 2006 and May 2009. The clinical results of improvement in pain and neurologic function were evaluated after an average of 25 months of follow-up, and changes on the imaging findings were evaluated. Preoperative and postoperative pain severity was assessed according to a $10-\mathrm{cm}$ VAS. Moreover, we also analyzed the postoperative complications.

RESULTS: Most patients experienced some degree of pain relief and functional improvement after fibrin glue therapy, with most experiencing complete or marked resolution of clinical symptoms. Twenty-one patients (55.3\%) reported excellent recovery, 12 (31.5\%) reported good recovery, 4 (10.5\%) reported fair recovery, and 1 (2.6\%) reported poor recovery. The overall percentage of positive outcomes (excellent and good recovery) was $86.8 \%$. No serious postoperative complications were discovered.

concLusıoNS: CT-guided percutaneous injection of fibrin glue therapy is simple, safe, and effective for the management of symptomatic sacral arachnoid cysts.

ABBREVIATIONS: VAS $=$ Visual analog pain scale

S acral arachnoid cysts are collections of CSF covered by arachnoidal cells and collagen that occur in a perineural or extradural site of the sacral spinal canal. ${ }^{1-3}$ Because sacral arachnoid cysts often occur asymptomatically, the overall population prevalence is unknown. However, sacral arachnoid cysts are not uncommon in clinical practice, representing $1 \%-5 \%$ of patients in a series undergoing lumbosacral MR imaging. ${ }^{4,5}$ Although the cysts are typically asymptomatic, approximately $20 \%-30 \%$ of patients with arachnoid cysts have clinical symptoms. ${ }^{4}$ When arachnoid cysts occur in the sacral spinal canal, they may impinge on nerve roots or the sacral nerve plexus, causing lower back pain, sciatica, bowel and bladder dysfunction, and sexual disturbances.

Traditionally, symptomatic sacral arachnoid cysts have been treated with cyst aspiration, which offers temporary symptomatic relief but is rarely curative due to cyst re-inflation. ${ }^{5}$ Surgical excisions of the cyst, such as cyst wall resection or fulguration or complete cyst removal, are invasive and may be complicated by nerve injury, hemorrhages, CSF leaks, and recurrence of the cyst. ${ }^{2,6}$ More recently, Patel et $\mathrm{al}^{7}$ reported an improved method of managing these patients with sacral

Received November 23, 2010; accepted December 17.

From the Department of Orthopedics, Union Hospital, Tongji Medical College, Huazhong University of Science and Technology, Wuhan, China.

Both Zengwu Shao and Baichuan Wang are first authors.

Please address correspondence to Zengwu Shao, MD, Department of Orthopedics, Union Hospital, Tongji Medical College, Huazhong University of Science and Technology, 1277 Jiefang Rd, Wuhan, 430022, China; e-mail express: SZWpro@163.com

Indicates open access to non-subscribers at www.ajnr.org

http://dx.doi.org/10.3174/ajnr.A2595 arachnoid cysts with severe clinical symptoms. The procedure involves cyst aspiration and placement of fibrin glue into the cyst cavity with CT guidance. Thirty-eight patients with symptomatic sacral arachnoid cysts underwent fibrin glue therapy in our hospital between June 2006 and May 2009 to determine whether injection of fibrin glue into an arachnoid cyst after aspiration of CSF offers a safe and effective therapy.

\section{Materials and Methods}

\section{Clinical Characteristics}

Thirty-eight patients with symptomatic sacral arachnoid cysts (18 men, 20 women; mean age, 37.6 years; range, 24-68 years; mean disease duration, 18.2 months; range, 9-72 months) were enrolled in this study. Of the 38 patients, 20 were from a pain clinic, 5 were from a neurologic clinic, and 13 were from an orthopedic clinic. Clinical manifestations of these patients included lumbosacral pain, sciatica, perineum/saddle and leg discomfort, bowel and bladder dysfunction, and muscle atrophy (Table 1). Twenty-one patients experienced lumbosacral pain symptoms, accompanied by numbness of the perineal region or lateral posterior aspect of the legs. Eleven patients had pain in the legs (sciatica) with or without muscle atrophy. Six patients only exhibited symptoms of cauda equina compression with sensory abnormalities in the perineum/saddle area, bladder and bowel dysfunction, or a burning sensation around the anus. The symptoms of 12 patients were associated with postural change. Standing or walking worsened the pain and numbness, while bed rest significantly alleviated these symptoms.

\section{Radiographic Signs}

All patients underwent preoperative x-ray, CT, and MR imaging, except for 2 patients who had stents for the treatment of coronary heart 
Table 1: Summary of cyst characteristics and clinical symptoms in 38 patients

\begin{tabular}{|c|c|c|}
\hline $\begin{array}{l}\text { Cyst } \\
\text { Location }\end{array}$ & $\begin{array}{l}\text { No. of } \\
\text { Cysts }\end{array}$ & Clinical Symptoms \\
\hline L5-S1 & $11(28.9 \%)^{a}$ & $\begin{array}{c}\text { Leg pain, numbness in perineal region } \\
\text { and legs, muscle atrophy }\end{array}$ \\
\hline S1-S2 & $14(36.8 \%)^{a}$ & $\begin{array}{l}\text { Lumbosacral pain, constipation, urinary } \\
\text { frequency, and perineal discomfort } \\
\text { (numbness) }\end{array}$ \\
\hline S2-S3 & $15(39.5 \%)$ & $\begin{array}{l}\text { Lumbosacral pain, constipation, urinary } \\
\text { frequency and burning sensation } \\
\text { around the anus }\end{array}$ \\
\hline
\end{tabular}

${ }^{a}$ Two large cysts occupy 2 segments (L5-S1 and S1-S2).

disease for whom MR imaging was contraindicated. On neuroimaging examination, 25 of 38 patients had a single cyst and the remaining 13 patients had multiple cysts. The location of these cysts varied from L5 to S3 vertebral segments, with 9 in L5-S1, 12 in S1-S2, 15 in S2-S3, and 2 in L5-S2 (Table 1). To exclude urologic, anorectal, and orthopaedic diseases, we took these patients' medical histories and performed a variety of physical examinations related to patients' symptoms in detail. On the basis of patients' symptoms and positive physical signs (such as local lumbosacral percussion pain, decreased muscle strength of the lower extremity, and sensory abnormalities in the perineum or legs) and imaging findings (a large cyst out of the arachnoid mater on MR/CT imaging), we confirmed that the clinical symptoms were the result of these cysts.

\section{Treatment Protocol}

The study was approved and monitored by the Human Research Ethical Committee of Union Hospital, Tongji Medical College, Huazhong University of Science and Technology. Moreover, all patients were allowed to weigh the risks and benefits of fibrin glue therapy as a new method before signing informed consent.

Patients were placed prone on the CT table for the entire procedure. The cysts were located by using $1.5-\mathrm{mm}$-thick contiguous CT scans. To determine the location, depth, and angle of needle entry, we drew a diagram as shown in Fig $1 A$. An 11-ga bone-puncture needle (Fig $1 E$ ) was inserted into the cyst according to the location, angle, and depth determined as previously described after local anesthesia with $1 \%$ lidocaine. Intraoperative CT scans demonstrated a metal needle shadow within the cavity, confirming that the puncture needle was inserted into the cyst (Fig 1C). The values of the CT scans in Hounsfield units were recorded before and after injection of fibrin glue to confirm fibrin glue placement into the cyst cavity (Fig $1 B$ and $D$; before versus after, $10.9 \pm 3.5$ versus $49.8 \pm 8.6$; $P<.001$ ). The CSF was slowly aspirated to prevent pain and discomfort caused by sudden decompression.

After percutaneous drainage of the sacral cyst, fibrin glue was injected into the cavity of the cyst. The fibrin glue was used according to the manufacturer's instructions (PSW; Shanghai RAAS Blood Products, Minhang, Shanghai, China). Fibrin glue is composed of a main glue, containing fibrinogen dissolved in a phosphate buffer solution, and a thrombin catalyst dissolved in a calcium chloride solution. The main glue and catalyst were loaded separately into 2 syringes
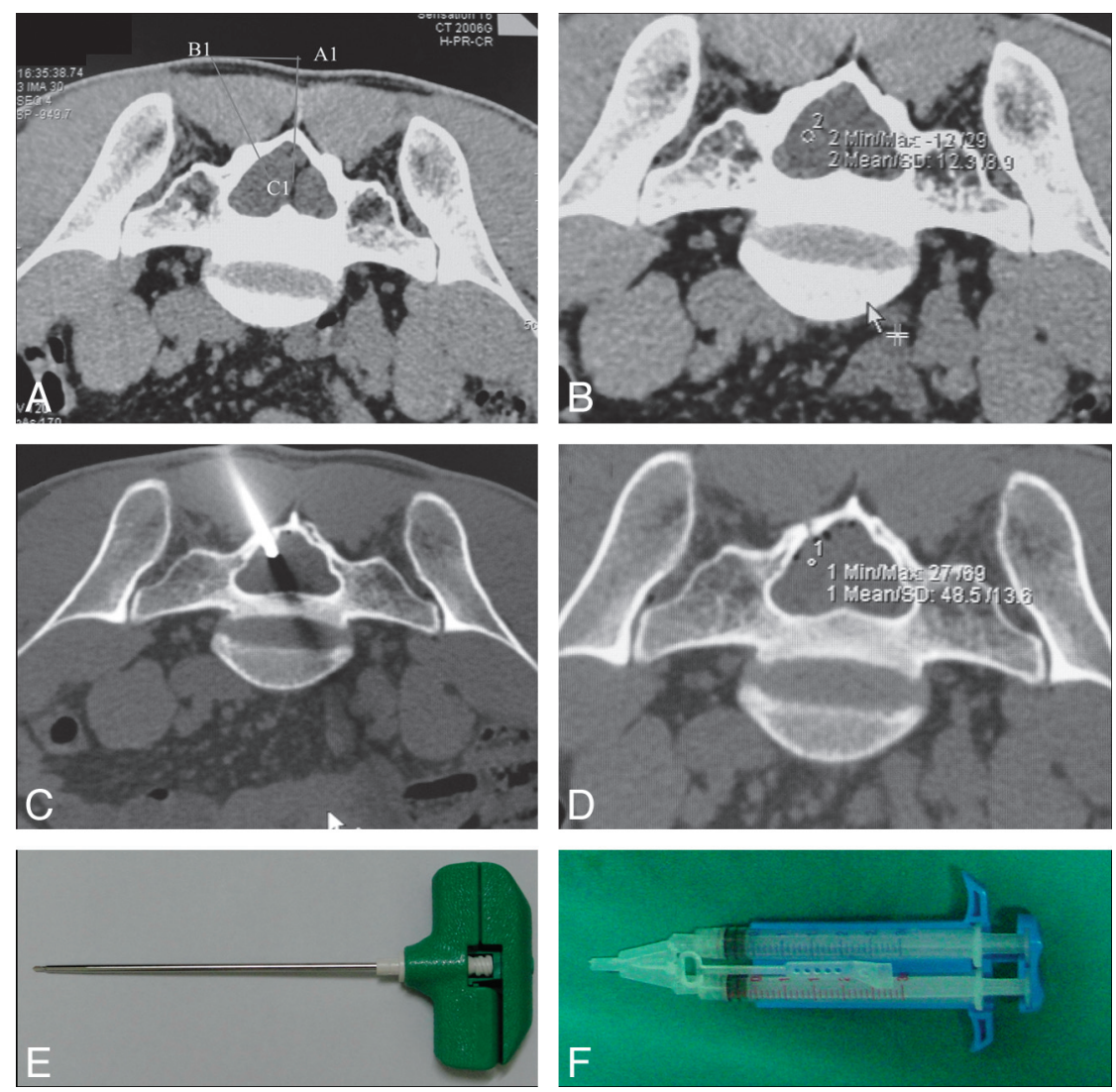

Fig 1. $A$, Depth and angle of the puncture needle entry. Neuroimages demonstrate CT-guided percutaneous injection of fibrin glue. $B$, Preoperative CT scans demonstrate a collection of CSF (CT value $=12.3$ ) within the cyst. $C$, Intraoperative CT scans demonstrate the needle placement into the center of the cyst. $D$, Postoperative CT scans demonstrate hyperattenuated material (CT value $=48.5$ ) within the cyst, consistent with aggregated fibrin glue. The CT values before and after fibrin glue placement are shown in $B$ and $D$. $E-F$, Pictures of puncture needle $(E)$ and syringe pump system $(F)$ consisting of 2 syringes connected via a connecting stent. 
Table 2: Recovery from lumbosacral pain and sciatica after a mean follow-up of 25 months in 38 patients ${ }^{\text {a }}$

\begin{tabular}{lccccc}
\hline & & & \multicolumn{2}{c}{ No. of Patients } \\
\cline { 2 - 5 } Intervention & Painless & Mild Pain & Moderate Pain & Severe Pain \\
\hline Preoperative & 6 & 2 & 16 & 14 \\
Postoperative & 21 & 10 & 5 & 38 \\
\hline
\end{tabular}

a Recovery was assessed by the VAS (VAS of 0 , painless; 1-3, mild pain; 4-6, moderate pain; and 7-10, severe pain). Paired-samples $t$ test showed a significant difference between the preoperative and postoperative periods $(P<.001)$, suggesting that the fibrin glue therapy could significantly improve the symptoms of pain.

that were connected via a connecting stent (Fig $1 F)$. Equal volumes of main glue and catalyst solutions were injected simultaneously into the cyst cavity until the total volume was equal to the amount of fluid aspirated from the cyst. Although 13 patients had multiple cysts in this study, these individuals only underwent fibrin glue therapy of 1 or 2 cysts that were considered to be the main source of symptoms.

\section{Postoperative Care}

All patients were carefully monitored to detect any adverse reactions in time, postoperatively. An average of 3 days (range, 1-5 days) of bed rest was necessary for patients with postoperative discomfort (such as pain in the puncture site, pain from lumbosacral swelling, headache, and nausea and vomiting). Moreover, patients who had side effects of fibrin glue therapy (headache, fever, and nausea and vomiting) received 1 day of $250 \mathrm{~mL}$ of $20 \%$ mannitol and $5 \mathrm{mg}$ of dexamethasone, and 3 days of prophylactic antibiotics.

\section{Efficacy Evaluation}

The efficacy of the procedure was assessed quantitatively by the changes in pain scores and qualitatively by symptomatic and functional improvement before and after fibrin glue therapy. The assessment criteria, adopted from Zhang et al, ${ }^{8}$ were as follows: excellent, complete resolution of signs and symptoms, with the patient returning to his or her regular employment and no recurrence of cysts during $\geq 1$ year of follow-up; good, symptoms and signs in the legs and perineal region resolved but with persistent pain in the lumbosacral region, which did not interfere with the patient's regular work (the cysts did not recur for $\geq 6$ months during follow-up); fair, no improvement in clinical symptoms, but a decrease in cyst size on the imaging study; poor, no improvement in clinical symptoms and no observed changes in cyst size in imaging studies or recurrence. The preoperative and postoperative pain severity was assessed according to a $10-\mathrm{cm}$ VAS. Comparisons between preoperative and postoperative results were made by using a paired-samples $t$ test (Statistical Package for the Social Sciences, Version 11.0; SPSS, Chicago, Illinois).

\section{Results}

\section{Follow-Up Evaluation}

All patients were observed for 12-36 months with an average follow-up of 25 months. In this study, we especially selected a doctor who was not on our research team to perform the clinical assessment. Most patients experienced some alleviation of clinical symptoms (pain and neurologic dysfunction) after fibrin glue therapy (Table 2). Twentyone patients who experienced complete resolution of symptoms and had no recurrences evaluated their recovery as excellent $(55.3 \%)$. Twelve patients with partial resolution of symptoms and without cyst recurrence evaluated their recovery as good (31.5\%). Four patients without symptomatic improvement and with a decrease in cyst size rated their recovery as fair (10.5\%), and 1 patient with cyst recurrence
Table 3: Preoperative and postoperative size of cysts for all 38 consecutive patients after 3 months

\begin{tabular}{|c|c|c|}
\hline No. & Preoperative Size $\left(\mathrm{cm}^{3}\right)$ & Postoperative Size $\left(\mathrm{cm}^{3}\right)$ \\
\hline 1 & 13.5 & 0 \\
\hline 2 & 11.3 & 0 \\
\hline 3 & 15.6 & 6.3 \\
\hline 4 & 13.7 & 0 \\
\hline 5 & 11.8 & 0 \\
\hline 6 & 18.6 & 8.6 \\
\hline 7 & 23.2 & 9.5 \\
\hline 8 & 13.6 & 0 \\
\hline 9 & 11.8 & 0 \\
\hline 10 & 44.0 & 22.1 \\
\hline 11 & 18.6 & 0 \\
\hline 12 & 12.6 & 0 \\
\hline 13 & 18.6 & $15.8^{\mathrm{a}}(0)^{\mathrm{b}}$ \\
\hline 14 & 15.5 & 0 \\
\hline 15 & 13.9 & 0 \\
\hline 16 & 26.7 & 7.5 \\
\hline 17 & 19.8 & 0 \\
\hline 18 & 32.7 & $27.6^{\mathrm{a}}$ \\
\hline 19 & 18.3 & 0 \\
\hline 20 & 17.0 & 0 \\
\hline 21 & 25.7 & 9.3 \\
\hline 22 & 25.3 & 8.2 \\
\hline 23 & 16.8 & 0 \\
\hline 24 & 11.5 & 0 \\
\hline 25 & 19.3 & 9.7 \\
\hline 26 & 21.5 & 0 \\
\hline 27 & 22.8 & 9.6 \\
\hline 28 & 32.1 & $27.9^{a}(9.1)^{b}$ \\
\hline 29 & 52.7 & $52.8^{\mathrm{a}}$ \\
\hline 30 & 17.5 & 0 \\
\hline 31 & 13.2 & 3.1 \\
\hline 32 & 15.4 & 0 \\
\hline 33 & 16.9 & 0 \\
\hline 34 & 25.2 & 7.1 \\
\hline 35 & 28.3 & 9.2 \\
\hline 36 & 19.5 & 0 \\
\hline 37 & 15.2 & $28.3^{\mathrm{a}}(8.2)^{\mathrm{b}}$ \\
\hline 38 & 16.9 & 0 \\
\hline
\end{tabular}

${ }^{a}$ No significant changes in cyst size on MR imaging/CT studies obtained 3 months postoperatively

${ }^{b}$ Follow-up results performed at 3 months after a second fibrin glue therapy $(P<.001$, preoperative vs postoperative), suggesting that fibrin glue could effectively fill and close the cyst cavity.

rated her recovery as poor $(2.6 \%)$. The overall rate of positive outcomes (excellent and good recoveries) was $86.8 \%$. Follow-up MR imaging studies performed at 3 months after fibrin glue therapy showed that 21 cysts had completely disappeared and 12 cysts had significantly decreased in size (Table 3), and these disappeared and shrunken cysts did not re-inflate or increase in size during the follow-up interval (Fig 2). Moreover, we have observed 5 passable results (fair and poor recoveries) in this study. Three of these patients received fibrin glue therapy again and obtained satisfactory clinical outcomes. The other 2 patients rejected receiving 

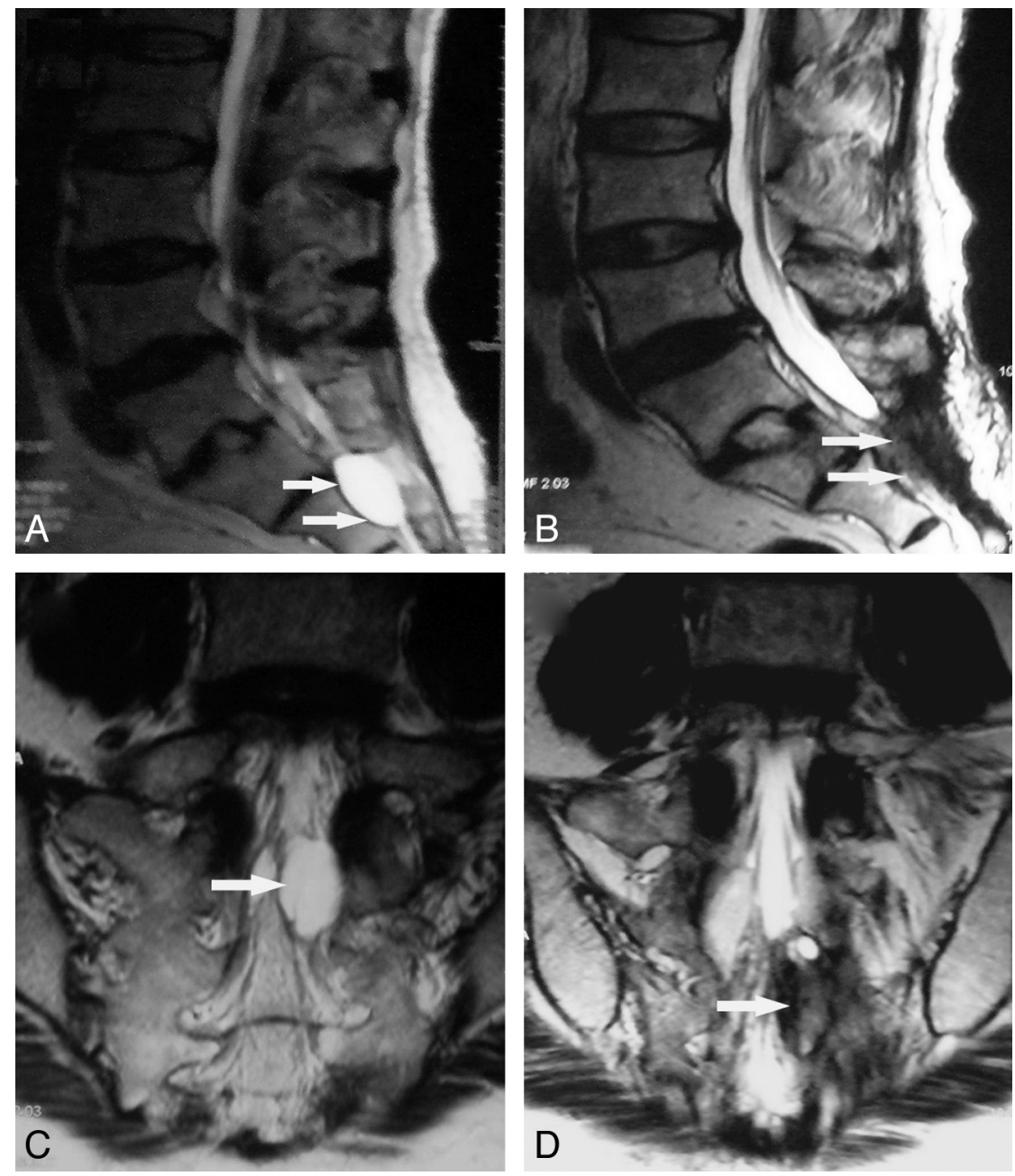

Fig 2. A 46-year-old woman who underwent percutaneous injection of fibrin glue with the aid of CT guidance for a symptomatic sacral arachnoid cyst. $A$ and $C$, Sagittal and coronal T2-weighted MR images before fibrin glue injection demonstrate that an expansile arachnoid cyst (arrow) is filling with CSF. B and D, Sagittal and coronal T2-weighted MR images obtained 7 months postoperatively demonstrate that the disappeared cyst (arrow) has not re-inflated with CSF.

fibrin glue therapy or surgical interventions because of many "unknown" reasons; they received conservative treatments with neurotrophic drugs and physical therapy.

\section{Postoperative Complications}

No cases were complicated by postoperative infection, nerve damage, or CSF leak. Five patients had headache, fever, and nausea and vomiting, which are side effects of fibrin glue therapy, at 1-2 days after the procedure. Two patients in whom the cyst was particularly large $(5.5 \times 2.5 \times 3.2 \mathrm{~cm}$ and $6.1 \times 3.2 \times$ $2.7 \mathrm{~cm})$ had moderate fever $\left(38.5^{\circ}-38.9^{\circ} \mathrm{C}\right)$ and mild neck stiffness (thought to result from a small amount of fibrin glue leaking into the CSF circulation) at 1 day postoperatively. These patients recovered completely after effective therapy: $1-3$ days of intravenous drip of 250-mL 20\% mannitol and 5-mg dexamathasone, and 3 days of prophylactic antibiotics.

\section{Discussion}

A great deal of confusion continues to exist about the precise definition of sacral arachnoid cysts. Sacral arachnoid cysts have been referred to as sacral meningeal cysts, sacral nerve root diverticula, sacral perineurial cysts, and sacral arachnoid pouches. $^{2,9-12}$ Although the sacral arachnoid cyst was first de- scribed by Tarlov in $1938,{ }^{2}$ the etiology of sacral arachnoid cysts remains unclear. Tarlov ${ }^{2}$ postulated that the cyst occurred as a result of an inflammatory process surrounding the nerve root sheath, followed by cyst formation. Nishiura et $\mathrm{al}^{9}$ described a history of antecedent trauma in $40 \%$ of their patients with sacral arachnoid cysts. On the contrary, most surgeons, including ourselves, consider the cysts to be a congenital arachnoidal defect. ${ }^{1,12-15}$ A postulated mechanism involves a potential or very small "ball-valve" communication (also called "traffic pore") between the congenital pouch (the cyst) and the subarachnoid space, by which CSF can flow into the pouch but not exit. Therefore, the cysts can enlarge progressively because of continuous 1-way infusion of CSF. Also, some researchers considered that these bulging pathologic entities are the result of simple outpouchings of the arachnoid, without a communication between the cyst and the subarachnoid space. ${ }^{10,16}$

Although often asymptomatic, enlarging sacral arachnoid cysts can cause significant discomfort. Symptoms related to a sacral arachnoid cyst arise from its compression or stretch of nerve root filaments around the lesion. Sacral arachnoid cysts have been reported to cause lumbosacral pain, sciatica, bowel and bladder dysfunction, and sexual disturbances. ${ }^{17-19}$ On 
rare occasions, these symptoms can be exacerbated by standing, coughing, and defecation, because elevated subarachnoid pressure forces CSF from the spinal subarachnoid space through a ball-valve-like communication into the perineurial cyst cavity. ${ }^{18,20}$ In addition to these subjective symptoms, sacral arachnoid cysts also cause various objective neurophysiologic abnormalities, including decreased sural nerve action potentials and sensory nerve conduction velocities. ${ }^{21,22}$

Sacral arachnoid cysts can be treated via a variety of methods. Surgical treatments include cyst resection at the neck, cyst wall resection or fulguration, cyst fenestration and imbrication, and complete cyst removal with excision of the affected nerve root. ${ }^{2,6,22-25}$ However, the main drawback of this surgical management is the high risk of recurrence and complications (such as meningitis, nerve damage, and CSF leak). More recently, a nonsurgical method with CT-guided percutaneous cyst drainage has been introduced as an alterative therapy for symptomatic sacral arachnoid cysts. ${ }^{5,6}$ Unfortunately, symptoms often recur after percutaneous cyst drainage because of recollection of CSF. Patel et $\mathrm{al}^{7}$ described an improved method that involves fibrin glue placement into the cyst cavity after CSF aspiration. Some follow-up MR imaging performed in patients who had undergone fibrin glue therapy revealed that the CSF had not collected again 4 months after the procedure. ${ }^{7}$

In this study, we performed the improved method in 38 patients with symptomatic sacral arachnoid cysts and conducted an average of 25 months of follow-up. In our follow-up evaluations, we found that 33 of our patients experienced some degree of pain relief and functional improvement after fibrin glue therapy, with most patients experiencing complete or marked resolution of clinical symptoms. Moreover, none of our patients had complications of postoperative infection, nerve damage, and CSF leak. Follow-up MR imaging in most patients revealed that the cyst had not re-accumulated CSF after fibrin glue therapy during the follow-up period. This success may be attributed to the gelatinous properties and biologic effects of fibrin glue. ${ }^{26}$ Fibrin glue can effectively fill the cyst cavity and seal the communication (traffic pore) between the cyst and the subarachnoid space, and subsequent fibroblast proliferation and fibrous scar formation that occurs with fibrin glue resorption to completely eliminate the cyst (Fig $2 B$ and $D)$.

Symptomatic sacral arachnoid cysts should be taken seriously by all neurosurgeons and orthopedic spine surgeons. For these symptomatic sacral arachnoid cysts, patients presenting with mild pain or sensory abnormalities should initially undergo medical treatments with anti-inflammatory drugs, neurotrophic drugs, and physical therapy. Fibrin glue therapy should be performed in patients with failed conservative treatments or severe pain and nerve dysfunction. Moreover, patients presenting with symptoms exacerbated by increased subarachnoid pressure are likely to benefit most from the fibrin glue therapy due to effectively blocking the communication between the cyst and the subarachnoid space after fibrin glue placement. Of course, a larger number of patients and a longer follow-up duration should be studied to further determine the efficacy of this procedure, which is our ongoing work.

\section{Conclusions}

Sacral arachnoid cyst is a congenital arachnoid defect. Although often asymptomatic, enlarging sacral arachnoid cysts can have serious complications and, therefore, should not be overlooked. In this study, a novel nonsurgical method with CT-guided percutaneous fibrin glue therapy was used to manage symptomatic sacral arachnoid cysts and obtained satisfactory clinical outcomes. Although our experience is very limited, we found that CT-guided fibrin glue therapy for symptomatic sacral arachnoid cysts is a simple, safe, and effective therapy.

\section{References}

1. Tarlov IM. Sacral Nerve Root Cysts: Another Cause of the Sciatica or Cauda Equina Syndrome. Springfield, Illinois: Charles C. Thomas; 1953:56-116

2. Tarlov IM. Perineural cysts of the spinal nerve roots. Arch Neurol Psychiatry 1938;40:1067-74

3. Howieson J, Norrell HA, Wilson CB. Expansion of the subarachnoid space in the lumbosacral region. Radiology 1968;90:488-92

4. Langdown AJ, Grundy JR, Birch NC. The clinical relevance of Tarlov cysts. J Spinal Disord Tech 2005;18:29-33

5. Paulsen RD, Call GA, Murtagh FR. Prevalence and percutaneous drainage of cysts of the sacral nerve root sheath (Tarlov cysts). AJNR Am J Neuroradiol 1994;15:293-97

6. Wilkins WH. Commentary on prevalence and percutaneous drainage of cysts of the sacral nerve root sheath (Tarlov cysts). AJNR Am J Neuroradiol 1994;15:298-99

7. Patel MR, Louie W, Rachlin J. Percutaneous fibrin glue therapy of meningeal cysts of the sacral spine. AJR Am J Roentgenol 1997;168:367-70

8. Zhang T, Li Z, Gong W, et al. Percutaneous fibrin glue therapy for meningeal cysts of the sacral spine with or without aspiration of the cerebrospinal fluid. J Neurosurg Spine 2007;7:145-50

9. Nishiura I, Koyama T, Handa J. Intrasacral perineurial cyst. Surg Neurol 1985;23:265-69

10. Rexed BA, Wennstrom K. Arachnoidal proliferation and cystic formation in the spinal nerve-root pouches of man. J Neurosurg 1959;16:73-84

11. Tarlov IM. Spinal perineurial and meningeal cysts. J Neurol Neurosurg Psychiatry 1970;33:833-43

12. Strully KJ. Meningeal diverticula of sacral nerve roots (perineural cysts) JAMA 1956;161:1147-52

13. Hoshino $\mathrm{Y}$, Edakuni $\mathrm{H}$, Shimada $\mathrm{H}$, et al. Sacral arachnoid cyst associated with Marfan syndrome. Intern Med 2005;44:271-73

14. Williams B. Sciatica caused by sacral-nerve-root cysts. Lancet 1971;1:137

15. Tanaka M, Nakaharab S, Ito Y, et al. Surgical results of sacral perineural (Tarlov) cysts. Acta Med Okayama 2006;60:65-70

16. Rexed B. Arachnoidal proliferations with cyst formation in human spinal nerve roots at their entry into the vertebral foramina: preliminary report. J Neurosurg 1947;4:414-21

17. Schreiber F. Haddad B. Lumbar and sacral cysts causing pain. J Neurosurg 1951;8:504-09

18. Mummaneni PV, Pitts LH, McCormack BM, et al. Microsurgical treatment of symptomatic sacral Tarlov cysts. Neurosurgery 2000;47:74-79

19. Elsberg CA, Dyke CG, Brewer ED. The symptoms and diagnosis of extradural cysts. Bull Neurol Inst NY 1934;3:395-417

20. Nathan H, Rosner S. Multiple meningeal diverticula and cysts associated with duplications of the sheaths of spinal nerve posterior roots. J Neurosurg 1977;47:68-72

21. Cattaneo L, Pavesi G, Mancia D. Sural nerve abnormalities in sacral perineural (Tarlov) cysts. J Neurol 2001;248:623-24

22. Yucesoy K, Naderi S, Ozer H, et al. Surgical treatment of sacral perineural cysts: a case report. Kobe J Med Sci 1999;45:245-50

23. Voyadzis JM, Bhargava P, Henderson FC. Tarlov cysts: a study of 10 cases with review of the literature. J Neurosurg 2001;95:25-32

24. Strully KJ, Heiser S. Lumbar and sacral cysts of meningeal origin. Radiology 1954;62:544-49

25. Caspar W, Papavero L, Nabhan A, et al. Microsurgical excision of symptomatic sacral perineurial cysts: a study of 15 cases. Surg Neurol 2003;59:101-06

26. Siedentop KH, Harris DM, Ham K, et al. Extended experimental and preliminary surgical findings with autologous fibrin tissue adhesive made from patients own blood. Laryngoscope 1986;96:1062-64 